\title{
CONDIÇÕES CRÔNICAS, MULTIMORBIDADE E POLIFARMÁCIA NA
} POPULAÇÃO ADULTA

\section{CHRONIC CONDITIONS, MULTIMORBIDITY AND POLYPHARMACY IN THE ADULT POPULATION}

\author{
Andrieli Lovatel ${ }^{1}$, Eduarda Koch ${ }^{1}$, Luciano Fiorentin ${ }^{2}$, Marcia Terezinha da Rocha Restelatto ${ }^{3}$, \\ Vilma Beltrame ${ }^{4}$
}

Graduanda de Enfermagem - Universidade do Oeste de Santa Catarina ${ }^{1}$; Mestrando em Biociências e Saúde Universidade do Oeste de Santa Catarina - Unoesc ${ }^{2}$; Mestre em Biociência e Saúde - Docente do Curso de Enfermagem da Universidade do Oeste de santa Catarina ${ }^{3}$ e Doutora em Gerontologia Biomédica - Docente do Programa de Mestrado Biociências e saúde da Universidade do Oeste de Santa Catarina ${ }^{4}$

\section{Abstract}

This study aims to describe the prevalence of chronic conditions, multimorbidity and polypharmacy in the adult population of a municipality in southern Brazil. It is a crosssectional community-based study, carried out with individuals aged 30 years or older living in the municipality of Joaçaba, SC. Statistical tests (chi-square and Fisher's Exact Test) were applied, considering significant differences when $p<0.05$. 376 individuals participated in the study, 55.3\% in the age group of 30 to 59 years old (non-elderly) and $44.7 \%$ with 60 years old or more (elderly); 72\% of participants reported a health problem, with the highest prevalence among the elderly ( $p$ $=0.001) ; 47 \%$ had multimorbidity, of which $31.1 \%$ were elderly ( $p=0.001$ ). Polypharmacy was identified in $20.4 \%$ of the participants and $15.1 \%$ were in the elderly age group ( $p=0.001)$. Thus, it was observed that chronic diseases are present in adults from 30 years of age and advancing age make multimorbidity and polypharmacy more evident.

Keywords: Chronic disease. Polymedication. Epidemiology. Adult. Old-ged.

\section{Resumo}

Esse estudo tem como objetivo descrever a prevalência de condições crônicas, multimorbidade e polifarmácia na população adulta de um município do Sul do Brasil. É um estudo transversal de base comunitária, realizado com indivíduos de 30 anos ou mais residentes no município de Joaçaba, SC. Foram aplicados testes estatísticos (qui-quadrado e Fisher'sExact Test), considerando-se diferenças significantes quando valor de $p<0,05$. Participaram do estudo 376 indivíduos, 55,3\% na faixa etária de 30 a 59 anos (não idoso) e 44,7\% com 60 anos ou mais (idoso); 72\% dos participantes referiam problema de saúde, sendo a maior prevalência entre os idosos ( $p=0,001) ; 47 \%$ apresentaram multimorbidade, desses $31,1 \%$ eram idosos $(p=0,001)$. A polifarmácia foi identificada em $20,4 \%$ dos participantes e 15,1\% eram da faixa etária idosa $(p=0,001)$. Assim, observou-se que as doenças crônicas estão presentes em adultos a partir de 30 anos e o avançar da idade tornam a multimorbidade e a polifarmácia mais evidentes.

Palavras Chave: Doença Crônica. Polimedicação. Epidemiologia. Adulto. Idoso. 
O envelhecimento populacional vem acarretando transformações na incidência e prevalência das doenças e consequentemente, aumentando os índices de perda de qualidade de vida e de mortes prematuras causadas pelas Doenças Crônicas Não Transmissíveis (DCNT) ${ }^{1,2}$. As DCNT's são as principais fontes de carga de doença no Brasil e responsáveis pela maioria das doenças e mortes, tanto em países de alta, média ou baixa condição socioeconômica. ${ }^{3,4}$

As DCNT's desenvolvem-se no decorrer da vida e são de longa duração. Nas últimas décadas passaram a liderar as causas de óbito no Brasil, ultrapassando os índices de mortalidade por doenças infecciosas e parasitárias da década de $80 .{ }^{5,6}$ No Brasil são responsáveis por $72,0 \%$ das causas de óbitos, com maior prevalência em idosos, porém cada vez mais os não idosos também são acometidos pelas doenças crônicas. 7

As principais condições crônicas prevalentes na população brasileira são as doenças cardiovasculares, doenças respiratórias, diabetes mellitus e neoplasias. Elas possuem quatro fatores em comum: tabagismo, uso nocivo do álcool, alimentação não saudável e sedentarismo. ${ }^{7}$

A coexistência de duas ou mais condições crônicas que afetam o indivíduo, é conhecida pelo termo multimorbidade, sem privilegiar essa ou aquela doença, ou ainda sem que uma delas possa ser considerada como problema principal. ${ }^{8,9}$

Vários estudos comprovam que a presença de multimorbidade aumenta com a idade, diante disso, a maioria das pesquisas são realizadas na população idosa. ${ }^{8-10}$ Entre as condições crônicas que mais atingem os idosos, destaca-se a Hipertensão Arterial e Diabetes Mellitus, que são consideradas como principais fatores de risco para o desenvolvimento de doenças cerebrovasculares, cardíacas e complicações renais. As doenças crônicas que também atingem os idosos, porém em menor proporção, são as neoplasias, as doenças mentais, respiratórias e inflamatórioreumáticas. $^{12}$

A prevalência de multimorbidade acarreta no maior uso de medicamentos e, o uso de 5 ou mais medicamentos por um indivíduo é denominado de polifarmácia. O aumento no uso de fármacos está relacionado com o aumento da expectativa de vida e o consequente aumento de multimorbidade. $^{13,14}$

O uso de associações de medicamentos, - qual muitas vezes atinge o número característico do uso de polifármacos, é uma forma encontrada para controlar as multimorbidades. Quando ocorre de forma otimizada, essa prática oportuniza o alcance da qualidade de vida. Porém, terapias prescritas e utilizadas de forma inadequada podem acarretar em efeitos danosos a saúde, indo na contramão da terapia esperada. ${ }^{14}$

Entre os motivos para o uso inapropriado de medicamentos estão os tratamentos não baseado em evidências, a prescrição simultânea por vários médicos, necessidade de uso de fármacos para amenizar efeitos secundários de outros medicamentos e a adoção de combinações medicamentosas para o sucesso terapêutico. As Combinações medicamentosas, possuem potenciais riscos de interações, assim surgindo novos problemas de saúde. ${ }^{15,16}$

É importante discutir ações para a promoção de uso racional de fármacos, educação continuada dos profissionais que prescrevem medicações e adoção de medidas para assistência farmacêutica, com o propósito da redução do uso inapropriado de fármacos. ${ }^{17}$

Conhecer o perfil epidemiológico da população em relação à prevalência das condições crônicas e de multimorbidade é fundamental para que se planeje as ações de prevenção e tratamento de forma efetiva, melhorando as condições de saúde e otimizando o custo da assistência.

Diante desse contexto, este estudo tem como objetivo identificar a prevalência de condições crônicas, multimorbidade e polifarmácia na população com 30 anos e mais na atenção básica do município de Joaçaba - SC.

\section{Método}

Estudo transversal de base comunitária, feito com indivíduos de 30 anos ou mais residentes na área de abrangência dos serviços de atenção básica do município de Joaçaba - SC, entre os dias 26 a 30 de novembro de 2018 e 7 e 9 de janeiro de 2019. 
Foram incluídos no estudo, adultos com 30 anos ou mais de idade, moradores na zona urbana do município na data da pesquisa. Como critério de exclusão considerou-se os indivíduos que, no momento da entrevista, estavam viajando, privados de liberdade por decisão judicial e os que estavam em internação hospitalar. A seleção da amostra foi realizada pelo método de amostragem por conglomerados, utilizando-se como unidade de referência para a localização da população as áreas mapeadas pelas Unidades de Estratégia Saúde da Família. Foram percorridos a quantidade necessária de domicílios, sempre se iniciando pela esquina da rua e seguindo o sentido horário. Todas as áreas existentes no município $(n=8)$ participaram do estudo, e a amostra foi composta por 47 indivíduos de cada área, totalizando 376 participantes.

Os dados foram coletados através de questionário estruturado para obter, além das variáveis que compõem os desfechos, as informações sobre faixa etária.

A coleta de dados foi feita por meio de entrevistas, realizadas nos domicílios dos participantes.

A análise dos dados foi realizada através de estatística descritiva e as variáveis foram expressas em número absoluto, média desvio padrão e percentuais, conforme a forma de apresentação. Para a comparação de variáveis foi usado o teste do Qui quadrado ou Fisher's Exact. As variáveis foram expressas por seus valores médios \pm desvio padrão quando apresentarem distribuição normal e, caso contrário, pela mediana e intervalo interquartil. Todas as diferenças foram consideradas diferentes quando $p<0,05$.

Os participantes foram classificados em faixa etária não idosa (de 30 a 59 anos) e faixa etária idosa (60 anos ou mais). Foram considerados portadores de multimorbidade os indivíduos que apresentaram 2 ou mais doenças crônicas, assim como, portador de polifarmácia os indivíduos que ingerem 5 ou mais medicamentos por dia.

O projeto de pesquisa foi aprovado pelo Comitê de Ética em Pesquisa, sob o parecer no 2.191.079.

\section{Resultados}

Participaram do estudo 376 pessoas, 55,3\% não idosa e 44,7\% idosa. Possuir condições crônicas foi referido por $72,1 \%$ dos participantes e foram classificação de 17 tipos diferentes. Os problemas de saúde de maior frequência entre a população estudada, foram a hipertensão arterial sistêmica com $42,3 \%$, problema na coluna com $21,0 \%$, e hipercolesterolemia com $18,9 \%$. (Tabela 1)

Tabela 1 - Presença de condições crônicas auto referidas, multimorbidade e polifarmácia da população adulta. Joaçaba - SC, 2019.

\begin{tabular}{|c|c|c|c|c|}
\hline \multirow[t]{2}{*}{ Variável } & \multicolumn{2}{|c|}{ Sim } & \multicolumn{2}{|c|}{ Não } \\
\hline & $\mathbf{N}$ & $\%$ & $\mathbf{N}$ & $\%$ \\
\hline Problemas de Saúde & 271 & 72,1 & 105 & 27,9 \\
\hline Hipertensão Arterial & 159 & 42,3 & 217 & 57,7 \\
\hline Problema de Coluna & 79 & 21,0 & 297 & 79,0 \\
\hline Hipercolesterolemia & 71 & 18,9 & 305 & 81,1 \\
\hline Outro & 61 & 16,2 & 315 & 83,8 \\
\hline Diabetes Mellitus & 57 & 15,2 & 319 & 84,8 \\
\hline Depressão & 53 & 14,1 & 323 & 85,9 \\
\hline Reumatismo & 50 & 13,3 & 326 & 86,7 \\
\hline Problema de Tireoide & 50 & 13,3 & 326 & 86,7 \\
\hline Problema de Coração & 43 & 11,4 & 333 & 88,6 \\
\hline Hipertrigliceridemia & 28 & 7,4 & 348 & 92,6 \\
\hline Problema de Audição & 19 & 5,1 & 357 & 94,9 \\
\hline Derrame/AVC & 16 & 4,3 & 360 & 95,7 \\
\hline Problema da Próstata & 13 & 3,5 & 104 & 27,7 \\
\hline Câncer & 10 & 2,7 & 366 & 97,3 \\
\hline Problema Renal & 8 & 2,1 & 368 & 97,9 \\
\hline Problema de Pulmão & 8 & 2,1 & 368 & 97,9 \\
\hline Total & & 376 & & 100 \\
\hline
\end{tabular}

Fonte: dados da pesquisa 
Conforme apresentado na Tabela 2, das $47,1 \%$ da população total da pesquisa que apresentam a multimorbidade, a maior prevalência deles, com 35,1\% referiram ter entre duas a quatro morbidades, seguidas por $9,8 \%$ com cinco a sete e $2,1 \%$ com oito ou mais.

Em relação a medicação, identificou-se que em 20,5\% dos participantes da pesquisa possuem polifarmácia, e 14,6\% tomam de 5 a 7 medicamentos ao dia.
Se considerarmos somente os participantes com polifarmácia $(20,5 \%)$ o índice de usuários de 5 a 7 medicamentos representa $71,43 \%$. Ressalta-se ainda que $29,8 \%$ dos participantes referiram não tomar nenhum tipo de medicamento. Sete pessoas $2,6 \%(n=7)$ das pessoas que referiram problemas crônicos de saúde, não referiram uso de medicamentos para a referida doença.

Tabela 2 - Presença de multimorbidade e polifarmácia da população adulta. Joaçaba - SC, 2019.

\begin{tabular}{lcc}
\hline \multicolumn{1}{c}{ Variável } & N & Porcentagem (\%) \\
\hline Multimorbidade & 177 & 47,1 \\
Sim & & \\
Quantidade de condições crônicas & 132 & 35,1 \\
De 2 a 4 & 37 & 9,8 \\
De 5 a 7 & 8 & 2,1 \\
8 ou mais & & \\
Polifarmácia & 77 & 20,5 \\
Sim & 299 & 79,5 \\
Não & & \\
Quantidade de medicamentos/dia & 55 & 14,6 \\
De 5 a 7 medicamentos & 22 & 5,9 \\
8 ou mais medicamentos & 376 & 100 \\
\hline Total & & \\
\hline Fonte: dados da pesquisa
\end{tabular}

Fonte: dados da pesquisa

A proporção de problemas de saúde entre a população está apresentada na tabela 3, onde $39,9 \%$ são pertencentes a faixa etária idosa e $32,2 \%$ na não idosos. Em relação as condições crônicas, a Hipertensão Arterial foi a mais referida, com $27,4 \%$ em idosos e $14,9 \%$ nos não idosos. Em seguida, e somando ambos os grupos, os problemas de coluna aparece com 21\%, hipercolesterolemia com $18,8 \%$ e reumatismo com $13,2 \%$.

As doenças crônicas foram referidas em ambas a faixa etária, entretanto foram mais frequentes entre os idosos. A exceção ocorreu no problema de próstata, referido somente em 3,5\% dos participantes idosos.

Tabela 3 - Presença de condições crônicas auto referidas, multimorbidades e polifarmácia da população idosa e não idosa. Joaçaba - SC, 2019

\begin{tabular}{|c|c|c|c|c|c|}
\hline \multirow[t]{2}{*}{ Variável } & \multicolumn{2}{|c|}{ Idoso } & \multicolumn{2}{|c|}{ Não Idoso } & \multirow[t]{2}{*}{$\mathbf{P}$} \\
\hline & $N=168$ & $(44,7 \%)$ & $N=208$ & $(55,4 \%)$ & \\
\hline \multicolumn{6}{|l|}{ Problemas de Saúde } \\
\hline Sim & 150 & $(39,9 \%)$ & 121 & $(32,2 \%)$ & \\
\hline Não & 18 & $(4,8 \%)$ & 87 & $(23,1 \%)$ & 0,01 \\
\hline \multicolumn{6}{|l|}{ Cipertensããấcterial } \\
\hline Não & 65 & $(17,3 \%)$ & 152 & (40.4\%) & 0,01 \\
\hline \multicolumn{6}{|l|}{ Problema de Coluna } \\
\hline $\operatorname{sim}$ & 51 & $(13,6 \%)$ & 28 & $(7,4 \%)$ & 0,01 \\
\hline Não & 117 & $(31,1 \%)$ & 180 & $(47,9 \%)$ & \\
\hline \multicolumn{6}{|l|}{ Hipercolesterolemia } \\
\hline Sim & 46 & $(12,2 \%)$ & 25 & $(6,6 \%)$ & \\
\hline Não & 122 & $(32,5 \%)$ & 183 & $(48,7 \%)$ & 0,01 \\
\hline \multicolumn{6}{|l|}{ Reumatismo } \\
\hline $\operatorname{sim}$ & 37 & $(9,8 \%)$ & 13 & $(3,4 \%)$ & \\
\hline Não & 131 & $(34,9 \%)$ & 195 & $(51,9 \%)$ & 0,01 \\
\hline
\end{tabular}


Continuação.

䓍

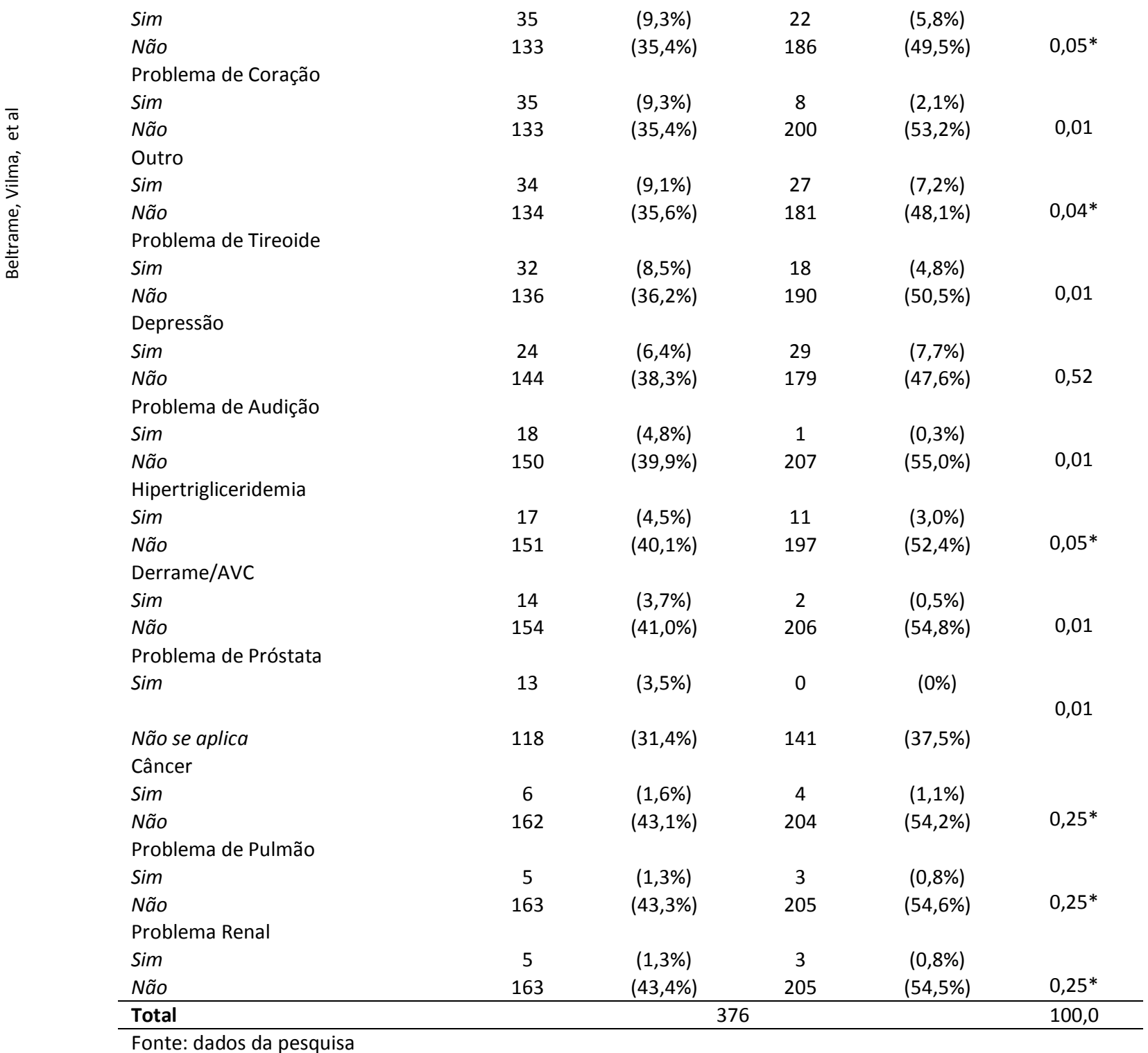

Em relação a quantidade de doenças crônicas e o uso de medicação contínua e a comparação entre os grupos de idosos e não idosos, a tabela 04 mostra que houve variação entre pessoas que referiram uma a mais de oito condições crônicas, independente do grupo de idosos ou não idosos. Entretanto, a multimorbidade está presente em $31,1 \%$ dos idosos $15,9 \%$ entre os não idosos $(p=0,01)$.

Quanto ao número de condições crônicas que as pessoas referiram possuir, a maior expressão esteve entre 2 a 4, com 45,8\% e $28,8 \%$ nos idosos e não idosos respectivamente $(p=0,01)$. 


\begin{tabular}{|c|c|c|c|c|c|}
\hline \multirow[t]{2}{*}{ Variável } & \multicolumn{2}{|c|}{ Idoso } & \multicolumn{2}{|c|}{ Não Idoso } & \multirow[t]{2}{*}{$\mathbf{P}$} \\
\hline & $N=168$ & $(44,7 \%)$ & $N=208$ & $(55,4 \%)$ & \\
\hline \multicolumn{6}{|l|}{ Multimorbidade } \\
\hline Sim & 117 & $(31,1 \%)$ & 60 & $(15,9 \%)$ & \\
\hline Não & 51 & $(13,6 \%)$ & 148 & $(39,4 \%)$ & $0,01^{*}$ \\
\hline \multicolumn{6}{|c|}{ Quantidade de Condições Crônicas } \\
\hline De 2 a 4 & 81 & $(45,8 \%)$ & 51 & $(28,8 \%)$ & \\
\hline De 5 a 7 & 31 & $(17,5 \%)$ & 6 & $(3,4 \%)$ & \\
\hline 8 ou mais & 5 & $(2,8 \%)$ & 3 & $(1,7 \%)$ & 0,01 \\
\hline \multicolumn{6}{|l|}{ Polifarmácia } \\
\hline Sim & 57 & $(15,1 \%)$ & 20 & $(5,3 \%)$ & \\
\hline Não & 111 & $(29,5 \%)$ & 188 & $(50 \%)$ & $0,01^{*}$ \\
\hline \multicolumn{6}{|c|}{ Quantidade de medicamentos dia } \\
\hline De 5 a 7 medicamentos & 43 & $(55,8 \%)$ & 12 & $(15,6 \%)$ & \\
\hline \multirow[t]{2}{*}{8 ou mais medicamentos } & 14 & $(18,2 \%)$ & 8 & $(10,4 \%)$ & \\
\hline & & & & & 0,01 \\
\hline
\end{tabular}

Fonte: dados da pesquisa

Em relação ao uso medicamento, $71,9 \%$ dos participantes referiram fazer uso de medicação contínua, sendo $41,4 \%$ idosos e $30,5 \%$ não idosos. Dentre os entrevistados, $15,1 \%$ dos idosos e $5,3 \%$ dos não idosos, possuem polifarmácia $(p=0,01)$. Desses, os que fazem uso de 5 a 7 medicamentos representam $55,8 \%$ e $15,6 \%$ e 8 ou mais medicamentos $18,2 \%$ e $10,4 \%$ são idosos e não idosos respectivamente.

\section{Discussão}

O resultado desse estudo apresentou que $71,2 \%$ dos participantes possuem pelo menos uma condição crônica de saúde, com maior significância no grupo de idosos $(p=0,01)$. Com o avançar da idade, esse fator passa a representar um indicador de tendência progressiva para o desenvolvimento de doenças cônicas. $^{18}$

Caracterizada pela presença de duas ou mais condições crônicas, a multimorbidades foi constatada em $47,1 \%$ dos participantes em geral, sendo que o percentual de $60,1 \%$ está entre o grupo de idosos, indo de encontro com o estudo de Barnett et al. ${ }^{19}$, realizado na Escócia em 2007, onde foi analisado banco de dados de 314 cartórios médicos e os dados de morbidades de
1.751.841 pessoas, constatou-se que mais da metade da população idosa, possuía duas ou mais doenças, e o maior percentual se deu nas idades entre 65 a 84 anos com $64,9 \%$ das multimorbidades.

Observa-se que o fato de haver prevalência de multimorbidades, ou mesmo, a presença significativa de um problema crônico de saúde em adultos não idosos, as tendências de possuir multimorbidade quando idosos, aumenta19, podendo alcançar índices de frequências semelhantes ao apresentado por Nunes, Thumé e Facchini que apresentou presença de multimorbidade em 4 para cada 5 pessoas idosas. $^{20}$

Das morbidades apresentadas, a hipertensão arterial (42,3\%), problema de coluna (21\%) e hipercolesterolemia (18,9\%), foram as doenças mais autorreferidas entre os participantes. Dado compatível com estudo transversal em uma coorte de base nacional da população brasileira não institucionalizada, com 50 anos ou mais entre os anos de 2015 e 2016, onde essas três doenças apareceram como destaque em pessoas acima de 50 anos.21 Também no estudo de base populacional realizada em 2008 com 1.593 idosos, a 
hipertensão é a condição crônica mais expressiva, identificada em 55,3\% dos idosos, seguidos por doenças de coluna com $37,4 \%{ }^{20}$

Dos participantes que possuem multimorbidade, $74,6 \%$ possuem de 2 a 4 condições crônicas. Desses 63\% faziam parte do grupo dos idosos e $37 \%$ no não idosos. Essa realidade confirma a estimativa do Brasil, que possui uma estimativa de que aproximadamente 26 milhões de pessoas com 50 anos ou mais possuem 2 ou mais condições crônicas e 18 milhões possuem 3 ou mais. ${ }^{21}$

A presença de multimorbidade no indivíduo gera duas consequências, o maior uso de medicamentos e também a maior procura pelos serviços de saúde ${ }^{13,22}$. Ao relacionarmos a multimorbidade com a polifarmácia, observa-se que $48,7 \%$ dos participantes idosos referiram uso de mais de cinco medicamentos ao dia. Essa proporção cai para 33,3\% no grupo dos não idosos. A multimorbidade possui uma forte associação com a polifarmácia. ${ }^{22}$

Dos $\quad 47 \% \quad$ participantes com multimorbidade, $20,4 \%$ usam mais de 5 medicamentos ao dia, que seria a polifarmácia, inferior ao percentual de $32 \%$ de prevalência de polifarmácia, encontrado no estudo transversal de base populacional com amostra de 1.705 idosos $^{23}$, e também, abaixo do estimado no Brasil, onde dos $70 \%$ dos idosos que possuem pelo menos uma patologia, $56 \%$ utilizam mais de quatro medicamentos contínuos como estratégia terapêutica. $^{24}$

Talvez isso se dê porque em várias condições crônicas, a terapêutica inicial pode ser conduzida conjuntamente e ou somente com alternativas terapêuticas não medicamentosas, como mudanças no estilo de vida, hábitos alimentares saudáveis, realização de atividades físicas entre outros cuidados básicos, já previstos em protocolos do Ministério da Saúde. ${ }^{25,27}$

Porém, a polifarmácia nem sempre pode ser evitada, da mesma forma, não representa efetivamente um problema, pois devido ao envelhecimento da população brasileira o uso de drogas como tratamento e prevenção de eventos adversos muitas vezes se faz necessária. ${ }^{23,28}$ Isso explica a presença expressiva do percentual de $74,0 \%$ da população idoso que faz uso de mais de 5 medicamentos ao dia, em relação aos não idosos $(26,0 \%)$.

\section{Conclusão}

Nessa pesquisa foi possível concluir que $72 \%$ dos entrevistados referiram possuir alguma condição crônica de saúde. As doenças que apresentaram maior frequência, foram a hipertensão arterial, problema de coluna e hipercolesterolemia. A multimorbidade foi identificada por $47 \%$ dos participantes, tendo a variável de 2 a 4 condições crônicas a mais autorreferida. A presença de polifarmácia na população estudada foi evidenciada em 20,5\% dos pesquisados e desses $14,6 \%$ relataram utilizar de 5 a 7 medicamentos ao dia.

Com os resultados apresentados, evidencia-se a presença de doenças crônicas em pessoas ainda na idade de 30 anos. Esse fato aumenta a probabilidade de ter multimorbidade com a avançar da idade. A tendência de aumentar o número de condições crônicas com o decorrer dos anos, traz o alerta para a necessidade de implementar medidas preventivas e de promoção à saúde a fim de reduzir o acometimento de doenças crônicas.

Assim, recomenda-se à população, a adesão de estilo de vida saudável, evitando ou reduzindo o desenvolvimento de multimorbidades e consequentemente a necessidade de uso de polifarmácia. Aos profissionais da saúde, em especial equipes da Estratégia Saúde da Família, o desenvolvimento de ações de promoção a saúde e prevenção às complicações decorrentes das condições crônicas, permitindo a diminuição no número de morbidades e quando necessário a utilização de associações farmacológicas adequadas retardando complicações inerentes às doenças crônicas e polifarmácia e melhorando a qualidade de vida.

\section{Referências}

1. MOTA $R$ DA SM, OLIVEIRA MLMC, BATISTA EC. Qualidade de vida na velhice: uma reflexão teórica. Communitas [Internet]. 15 de junho de 2017 [citado 24 de dezembro de 2020];1(1):47-61. Disponível em: https://periodicos.ufac.br/index.php/COMMUNI TAS/article/view/1122

2. MALTA DC, SILVA MMA DA, MOURA L DE, MORAIS NETO OL DE. A implantação do Sistema de Vigilância de Doenças Crônicas Não Transmissíveis no Brasil, 2003 a 2015: alcances e 
desafios. Rev bras epidemiol [Internet]. dezembro de 2017 [citado 24 de dezembro de 2020];20(4):661-75. Disponível em: http://www.scielo.br/scielo.php?script=sci_artte $x t \& p i d=S 1415$

3. SCHMIDT MI, DUNCAN BB, AZEVEDO E SILVA G, MENEZES AM, MONTEIRO CA, BARRETO $S M$, et al. Doenças crônicas não transmissíveis no Brasil: carga e desafios atuais. Lancet. 4 de junho de 2011;377(9781):1949-61.
4. GOULART FA DE A. Doenças Crônicas Não Transmissíveis: Estratégias De Controle $\mathrm{E}$ Desafios E Para Os Sistemas De Saúde [Internet]. Brasília, DF: OPAS; 2011 [citado 24 de dezembro de 2020]. Disponível em: https://apsredes.org/pdf/Condicoes-

Cronicas_flavio1.pdf

5. SILVA LA, BÚRIGO MJO, FARIA FG DE. Análise da situação de saúde do catarinense [Internet]. Florianópolis: Secretaria de Estado da Saúde; 2012 [citado 24 de dezembro de 2020]. Disponível em: http://www.dive.sc.gov.br/conteudos/publicacoe s/estudos_pesquisas/Analise_da_Situacao_de_Sa ude_do_Catarinense.pdf

6. MALTA DC, MOURA L DE, PRADO RR DO, ESCALANTE JC, SCHMIDT MI, DUNCAN BB. Mortalidade por doenças crônicas não transmissíveis no Brasil e suas regiões, 2000 a 2011. Epidemiol Serv Saúde [Internet]. dezembro de 2014 [citado 24 de dezembro de 2020];23(4):599-608. Disponível em: http://scielo.iec.pa.gov.br/scielo.php?script=sci_ arttext\&pid=S1679-

49742014000400002\&lng=en\&nrm=iso\&tlng=en

7. BRAZIL, organizador. Plano de ações estratégicas para o enfrentamento das doenças crônicas não transmissíveis (DCNT) no Brasil: 2011-2022 [Internet]. 1a edição. Brasília, DF: Ministério da Saúde; 2011. 154 p. (Série B. Textos básicos de saúde). Disponível em: http://bvsms.saude.gov.br/bvs/publicacoes/plan o_acoes_enfrent_dcnt_2011.pdf

8. GORZONI ML, COSTA EF DE A, MENESES $M$ DO $C L$ DE, LINS CD. Comorbidade, Multimorbidade e Manifestações Atípicas das Doenças nos Idosos. "in". Tratado de geriatria e gerontologia. Freitas, E. V. de [Internet]. 3o ed. Rio de Janeiro: Grupo Gen - Guanabara Koogan; 2000 [citado 24 de dezembro de 2020]. 2360 p. Disponível em: https://ftramonmartins.files.wordpress.com/201 6/09/tratado-de-geriatria-e-gerontologia-3c2aaed.pdf

9. GNÄDINGER $M$, HERZIG L, CESCHI $A$, CONEN D, STAEHELIN A, ZOLLER M, et al. Chronic conditions and multimorbidity in a primary care population: a study in the Swiss Sentinel Surveillance Network (Sentinella). Int J Public Health [Internet]. dezembro de 2018 [citado 24 de dezembro de 2020];63(9):1017-26. Disponível em: http://link.springer.com/10.1007/s00038018-1114-6

10. NUNES BP, THUMÉ E, FACCHINI LA. Multimorbidity in older adults: magnitude and challenges for the Brazilian health system. BMC Public Health [Internet]. dezembro de 2015 [citado 24 de dezembro de 2020];15(1):1172. Disponível em: http://bmcpublichealth.biomedcentral.com/articl es/10.1186/s12889-015-2505-8

11. VALDERAS JM, MERCER SW, FORTIN M. Research on Patients with Multiple Health Conditions: Different Constructs, Different Views, One Voice. J Comorb [Internet]. 1o de janeiro de 2011 [citado 24 de dezembro de 2020];1(1):1-3. Disponível https://doi.org/10.15256/joc.2011.1.11

12. DUNCAN BB, CHOR D, AQUINO EML, BENSENOR IM, MILL JG, SCHMIDT MI, et al. Doenças crônicas não transmissíveis no Brasil: prioridade para enfrentamento e investigação. Rev Saúde Pública [Internet]. dezembro de 2012 [citado 24 de dezembro de 2020];46(suppl 1):126-34. Disponível em: http://www.scielo.br/scielo.php?script=sci_artte $x t \& p i d=S 0034-$ $89102012000700017 \&$ lng $=p t \&$ tlng=pt 13. NASCIMENTO RCRM DO, ÁLVARES J, GUERRA JUNIOR AA, GOMES IC, SILVEIRA MR, COSTA EA, et al. Polypharmacy: a challenge for the primary health care of the Brazilian Unified Health System. Rev saúde pública [Internet]. 22 de setembro de 2017 [citado 24 de dezembro de 2020];51(suppl.2). Disponível em: https://www.revistas.usp.br/rsp/article/view/13 9761

14. FREITAS BY DE, MANO ET, OLIVEIRA PA DE, OLIVEIRA CC DE, SILVA JA DA, RODRIGUES A, et al. AVALIAÇÃO DA POLIFARMÁCIA EM PACIENTE IDOSO COM MULTIMORBIDADE. Diálogos Interdisciplinares [Internet]. 24 de novembro de 2018 [citado 29 de dezembro de 2020];7(3):194-214. Disponível em: https://revistas.brazcubas.br/index.php/dialogos /article/view/453

15. BUSHARDT RL, MASSEY EB, SIMPSON TW, ARIAIL JC, SIMPSON KN. Polypharmacy: Misleading, but manageable. Clin Interv Aging [Internet]. junho de 2008 [citado 24 de dezembro de 2020];3(2):383-9. Disponível em: https://www.ncbi.nlm.nih.gov/pmc/articles/PMC 2546482/ 
16. ALMEIDA NA DE, REINERS AAO, AZEVEDO RC DE S, SILVA AMC DA, CARDOSO JDC, SOUZA LC DE. Prevalence of and factors associated with polypharmacy among elderly persons resident in the community. Rev bras geriatr gerontol [Internet]. fevereiro de 2017 [citado 24 de dezembro de 2020];20(1):138-48. Disponível em: http://www.scielo.br/scielo.php?script=sci_artte xt\&pid=S180998232017000100138\&Ing=en\&tIng=en

17. CAMPOS MO, NETO JFR, SILVEIRA MF, NEVES DMR, VILHENA JM, OLIVEIRA JF, et al. Impacto dos fatores de risco para doenças crônicas não transmissíveis na qualidade de vida. Ciênc saúde coletiva [Internet]. março de 2013 [citado 24 de dezembro de 2020];18(3):873-82. Disponível em: http://www.scielosp.org/scielo.php?script=sci_ar ttext\&pid=S1413-

81232013000800033\&lng=pt\&nrm=iso\&tlng=pt 18. BRISCHILIAR SCR, AGNOLO CMD, GRAVENA AAF, LOPES TCR, ROMEIRO TC, PELLOSO SM. Doenças Crônicas não Transmissíveis e Associação com Fatores de Risco. Rev Bras Cardio [Internet]. 2014 [citado 28 de dezembro de 2020];27(1):35-42. Disponível em:

http://www.onlineijcs.org/sumario/27/pdf/v27n 1a06.pdf

19. BARNETT $K$, MERCER SW, NORBURY $M$, WATT G, WYKE S, GUTHRIE B. Epidemiology of multimorbidity and implications for health care, research, and medical education: a crosssectional study. The Lancet [Internet]. 7 de julho de 2012 [citado 24 de dezembro de 2020];380(9836):37-43. Disponível em: https://www.thelancet.com/journals/lancet/artic le/PIIS0140-6736(12)60240-2/abstract

20. NUNES BP, THUMÉ E, FACCHINI LA. Multimorbidity in older adults: magnitude and challenges for the Brazilian health system. BMC Public Health [Internet]. 25 de novembro de 2015 [citado 28 de dezembro de 2020];15(1):1172. Disponível em: https://doi.org/10.1186/s12889015-2505-8

21. NUNES BP, BATISTA SRR, ANDRADE FB DE, JUNIOR PRB DE S, LIMA-COSTA MF, FACCHINI LA. Multimorbidity: The Brazilian Longitudinal Study of Aging (ELSI-Brazil). Rev saúde pública [Internet]. 2018 [citado 24 de dezembro de 2020];52(Suppl 2):10s-10s. Disponível em: http://www.revistas.usp.br/rsp/article/view/153 952

22. ONG SM, LIM YMF, SIVASAMPU S, KHOO EM. Variation of polypharmacy in older primary care attenders occurs at prescriber level. BMC
Geriatrics [Internet]. 23 de fevereiro de 2018 [citado 24 de dezembro de 2020];18(1):59. Disponível em: https://doi.org/10.1186/s12877018-0750-2

23. PEREIRA KG, PERES MA, IOP D, BOING $A C, B O I N G A F, A Z I Z M$, et al. Polifarmácia em idosos: um estudo de base populacional. Rev bras epidemiol [Internet]. junho de 2017 [citado 28 de dezembro de 2020]; 20:335-44. Disponível em:

https://www.scielosp.org/article/rbepid/2017.v2 On2/335-344/

24. STUCHI BP. Polifarmácia em idosos na atenção primária [Internet] [Especialização em Saúde da Família]. [Rio de Janeiro]: UNASUS UNIVERSIDADE DO ESTADO DO RIO DE JANEIRO; 2016. Disponível em: https://ares.unasus.gov.br/acervo/html/ARES/79 86/1/Bruno\%20Pereira\%20Stuchi.pdf

25. BRASIL $M$ da S. ENVELHECIMENTO E SAÚDE DA PESSOA IDOSA [Internet]. 10 ed. Brasília - DF: Ministério da Saúde; 2006. 192 p. (Cadernos de Atenção Básica). Disponível em: https://bvsms.saude.gov.br/bvs/publicacoes/evel hecimento_saude_pessoa_idosa.pdf

26. BRASIL M da S. Estratégia para o cuidado da pessoa com doença crônica [Internet]. Brasília: Ministério da Saúde; 2014. 162 p. (Cadernos de Atenção Básica,). Disponível em: http://bvsms.saude.gov.br/bvs/publicacoes/estra tegias_cuidado_pessoa_doenca_cronica_cab35.p df

27. MARQUES $P$ DE $P$, FRANCISCO PMSB, BACURAU AG DE M, RODRIGUES PS, MALTA DC, BARROS NF DE. Uso de Práticas Integrativas e Complementares por idosos: Pesquisa Nacional de Saúde 2013. Saúde debate [Internet]. 16 de novembro de 2020 [citado 28 de dezembro de 2020]; 44:845-56. Disponível em: https://scielosp.org/article/sdeb/2020.v44n126/ $845-856 / p t /$

28. ROZENFELD S, FONSECA MJM, ACURCIO FA. Drug utilization and polypharmacy among the elderly: a survey in Rio de Janeiro City, Brazil. Rev Panam Salud Publica [Internet]. janeiro de 2008 [citado 24 de dezembro de 2020; 23:34-43. Disponível em: https://www.scielosp.org/article/rpsp/2008.v23n $1 / 34-43 /$ en/ 
Endereço para Correspondência

Vilma Beltrame

Endereço: Rua Guilherme Helmuth Arendit,

$\mathrm{n}-235$

Bairro: Centro. Concórdia - SC.

CEP: 89700-138.

vilma.beltrame@unoesc.edu.br

Telefone: 49-999300220

Recebido em 30/01/2021

Aprovado em 09/03/2021

Publicado em 10/05/2021 